\title{
Defuzzification method for ranking fuzzy numbers based on centroids and maximizing and minimizing set
}

\section{PhaniBushan RaoPeddi ${ }^{*}$}

${ }^{a}$ Department of Mathematics, Institute of Technology, GITAM (Deemed to be University), Visakhapatnam, Andhra Pradesh, 530045, India

\begin{tabular}{l}
\hline C H R O N I C L E \\
\hline Article history: \\
Received November22, 2018 \\
Received in revised format: \\
December28, 2018 \\
Accepted May25, 2019 \\
Available online \\
May25, 2019 \\
\hline Keywords: \\
Fuzzy numbers \\
Centroids \\
Maximizing set \\
Minimizing set \\
Index of optimism \\
\hline
\end{tabular}

\begin{abstract}
A B S T R A C T
This paper proposes a new method on ranking fuzzy numbers through the process of defuzzification by using maximizing and minimizing set on the triangular fuzzy numbers formed from generalized trapezoidal fuzzy numbers. In this method, a total utility value of each fuzzy number is defined by considering two left and two right utility values along with decision maker's optimism which serves as a criterion for ranking fuzzy numbers and overcomes the limitations of Chen's (1985) [Chen, S. H. (1985). Ranking fuzzy numbers with maximizing set and minimizing set. Fuzzy sets and systems, 17(2), 113-129] ranking method.
\end{abstract}

(C) 2018 by the authors; licensee Growing Science, Canada.

\section{Introduction}

Ranking fuzzy numbers is an important tool in decision making, artificial intelligence, data analysis and applications. Since the inception of fuzzy set theory by (Zadeh, 1965) and the first paper on ranking fuzzy numbers by (Jain, 1978) different scholars offered various techniques for ranking fuzzy numbers by representing the ill-defined quantities as fuzzy sets. Thus several studies have proposed various methods for ranking fuzzy numbers developed by applying maximizing set and minimizing set of fuzzy numbers considered to be an important breakthrough in ranking of fuzzy numbers. To minimize the computational procedure, (Chen, 1985) proposed a method on ranking fuzzy numbers based on maximizing and minimising set and by using total utility value of fuzzy numbers and this method is adopted by several decision makers in practical applications. This method has some short comings such as, the method cannot rank fuzzy numbers having same total utility values and when $x_{\max }$. or $x_{\min }$ is changed.To overcome the shortcomings in (Chen, 1985) ranking method, a new method is proposed in this paper on ranking fuzzy numbers. The process of defuzzification uses the total utility values of the fuzzy numbers which serves as a criterion for ranking fuzzy numbers. To define the total utility value of a fuzzy number, a generalized trapezoidal fuzzy number is considered which is treated as a trapezoid and then it is divided into three parts namely a triangle, rectangle and triangle followed by joining their respective centroids to form a triangular fuzzy number. The concept of maximizing and minimizing set is applied on this triangular fuzzy number to define two left and two right utility values along with decision maker's optimistic attitude thus defining the total utility value of each generalized trapezoidal

* Corresponding author.

E-mail address: phanibushanrao.peddi@gitam.edu(P.B.R.Peddi)

C2019 by the authors; licensee Growing Science, Canada. doi: $10.5267 / \mathrm{j}$.dsl.2019.5.004 
fuzzy number. The rest of the paper is organized as follows. In Section 2, the basic concepts of fuzzy numbers are reviewed. In Sections 3, the shortcomings of (Chen, 1985) method are discussed by considering two numerical examples. In Section 4, the new ranking method is presented and few examples are dealt elaborately addressing the short comings of (Chen, 1998) method. In Section 5, a comparative study is made with other existing methods taken from literature and finally the conclusions are presented in Section 6.

\section{Fuzzy numbers}

In this Section, the basic definitions of fuzzy numbers taken from (Dubois and Prade, 1978) are presented in brief.

Definition 2.1: A fuzzy number is defined as a convex normalized fuzzy set $\tilde{\mathrm{A}}$ of universal set $U$ such that

(a) there exists exactly one $x_{m} \in U$ called the mean value of $\tilde{\mathrm{A}}$ such that $f_{\tilde{\mathrm{A}}}\left(x_{m}\right)=1$,

(b) $f_{\tilde{\mathrm{A}}}(x)$ is piecewise continuous.

Definition 2.2: A real fuzzy number $\tilde{\mathrm{A}}$ is a fuzzy subset of the real line $R$ with membership function $f_{\tilde{\mathrm{A}}}(x)$ possessing the following properties:

(i) $f_{\tilde{\mathrm{A}}}(x)$ is a continuous mapping fromto the closed interval $[0, w], 0 \leq w \leq 1$,

(ii) $f_{\tilde{\mathrm{A}}}(x)=0$, for all $x \in(-\infty, a] \cup[d, \infty)$,

(iii) $f_{\tilde{\mathrm{A}}}(x)$ is strictly increasing on $[a, b]$ and strictly decreasing on $[c, d]$,

(iv) $f_{\tilde{\mathrm{A}}}(x)=w$, for all $x \in[b, c], w$ is a constant and $0 \leq w \leq 1$.

Here $a, b, c, d$ are real numbers and it is assumed that $\tilde{\mathrm{A}}$ is convex and bounded (i.e. $-\infty<a, d<\infty$ ). If $w=1$ in (iv), $\tilde{\mathrm{A}}$ is a normal fuzzy number, and if $0<w<1$ in (iv), $\tilde{\mathrm{A}}$ is a non-normal fuzzy number. The membership function $f_{\sim}$ of the real fuzzy number $\tilde{\mathrm{A}}$ (Fig. 1) is given by

$$
f_{\tilde{\mathrm{A}}}(x)= \begin{cases}f_{\tilde{\mathrm{A}}}^{L}(x), & a \leq x \leq b, \\ w, & b \leq x \leq c \\ f_{\tilde{\mathrm{A}}}^{R}(x), & c \leq x \leq d, \\ 0, & \text { otherwise }\end{cases}
$$

where $f_{\tilde{\mathrm{A}}}^{L}:[a, b] \rightarrow[0, w]$ is continuous, strictly increasing function and $f_{\tilde{\mathrm{A}}}^{R}:[c, d] \rightarrow[0, w]$ is continuous, strictly decreasing function.

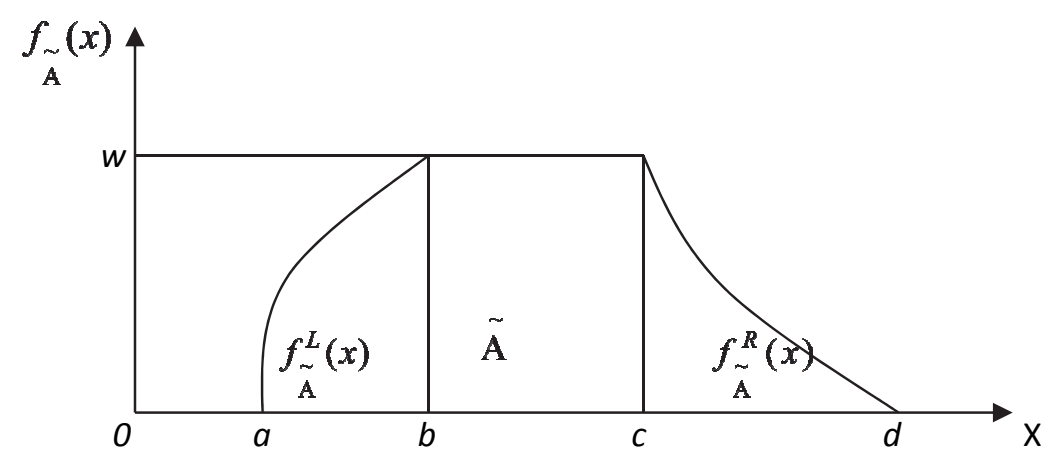

Fig.1: Fuzzy Number 


\section{Definition2.3: Trapezoidal fuzzy number}

If the membership function $f_{\widetilde{\mathrm{A}}}$ is piecewise linear, then $\tilde{\mathrm{A}}$ is said to be a trapezoidal fuzzy number. The membership function $f_{\sim}$ of a generalized or non-normal trapezoidal fuzzy number as shown in Fig. 2 is given by

$$
f_{\tilde{\mathrm{A}}}(x)= \begin{cases}\frac{w(x-a)}{b-a}, & a \leq x \leq b, \\ w, & b \leq x \leq c, \\ \frac{w(x-d)}{c-d}, & c \leq x \leq d, \\ 0, & \text { otherwise. }\end{cases}
$$

where $0 \leq w \leq 1$ and $a \leq b \leq c \leq d \in R$. A trapezoidal fuzzy number can be simply represented as $\tilde{\mathrm{A}}=(a, b, c, d ; w)$ and its image as $-\tilde{\mathrm{A}}=(-d,-c,-b,-a ; w)$.

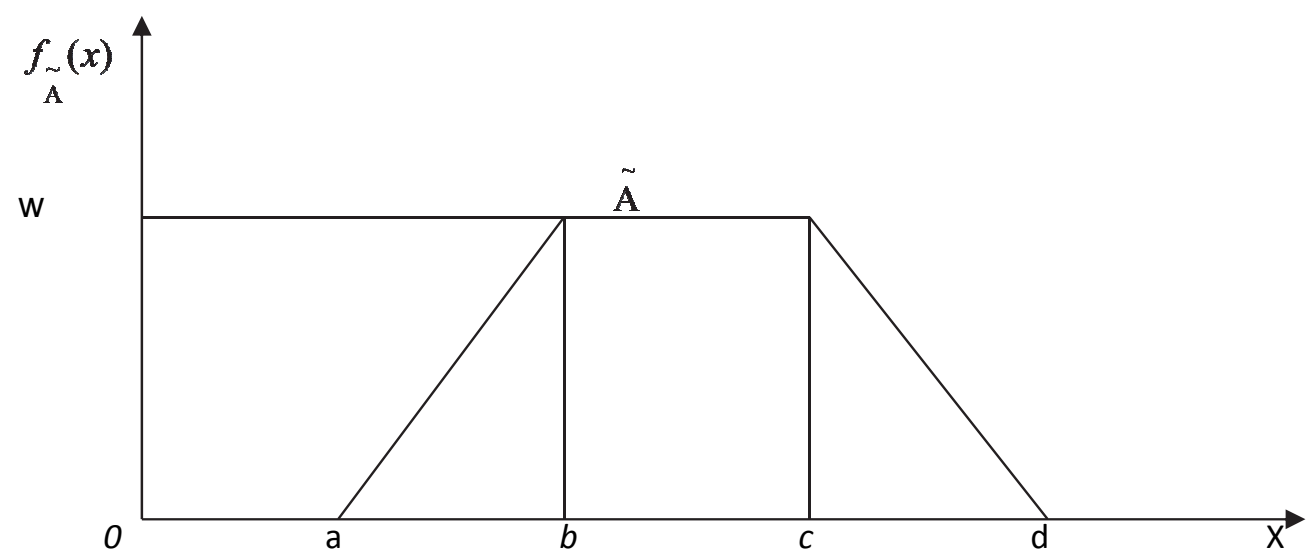

Fig. 2: Trapezoidal Fuzzy Number

As a particular case if $a<b=c<d$, the generalized trapezoidal fuzzy number reduces to a triangular fuzzy number given by $\tilde{\mathrm{A}}=(a, b, d ; w)$ where $0 \leq w \leq 1$. The value of ' $b$ ' corresponds to the mode or core and $[a, d]$ is the support of the triangular fuzzy number. If $w=1$, then $\tilde{\mathrm{A}}=(a, b, d)$ is called a normalized triangular fuzzy number. If $b \neq c$ then A is said to be a fuzzy interval or a flat fuzzy number and if $a=b=c=d$, then the fuzzy number $\tilde{\mathrm{A}}$ is said to be a crisp value.

\section{Shortcomings of (Chen, 1985) ranking method}

In (Chen, 1985) method, the total utility value of each fuzzy number $\tilde{\mathrm{A}}_{i}=\left(a_{i}, b_{i}, d_{i} ; w_{i}\right) ; 1 \leq i \leq n$ is calculated by the following:

$$
U_{J}(i)=\frac{w w_{i}}{2}\left[\frac{\left(d_{i}-x_{\min }\right)}{w_{i}\left(x_{\max }-x_{\min }\right)-w\left(b_{i}-d_{i}\right)}+\frac{1}{w_{i}}-\frac{\left(x_{\max }-a_{i}\right)}{w_{i}\left(x_{\max }-x_{\min }\right)+w\left(b_{i}-a_{i}\right)}\right]
$$


where $x_{\min }=\inf L, x_{\max }=\sup L, L=\bigcup_{i=1}^{n} L_{i}, L_{i}=\left\{x / f_{\tilde{\mathrm{A}}_{i}}(x)>0\right\}, w_{i}=\sup _{x} f_{\tilde{\mathrm{A}}_{i}}(x), w=\inf w_{i} . \quad$ This method is inconsistent and has led to some misapplications, namely the ranking outcome of fuzzy numbers changes when $x_{\max }$ or $x_{\min }$. is changed. These shortcomings are explained by the following examples:

\section{Example 3.1:}

Consider the following sets of fuzzy numbers:

Set 1: $\tilde{\mathrm{A}}_{1}=(3,5,7 ; 1), \tilde{\mathrm{A}}_{2}=\left(4,5, \frac{51}{8} ; 1\right), \tilde{\mathrm{A}}_{3}=(2,3,5 ; 1)$ and $\tilde{\mathrm{A}}_{4}=(8,9,10 ; 1)$.

Here $x_{\max }=10, x_{\min }=2$.

By using Eq. (1), the following are obtained

$U_{J}\left(\tilde{\mathrm{A}}_{1}\right)=\frac{1}{2}\left[\frac{(7-2)}{(10-2)-(5-7)}+1-\frac{(10-3)}{(10-2)+(5-3)}\right]=0.4$,

$U_{J}\left(\tilde{\mathrm{A}}_{2}\right)=\frac{1}{2}\left[\frac{\left(\frac{51}{8}-2\right)}{(10-2)-\left(5-\frac{51}{8}\right)}+1-\frac{(10-4)}{(10-2)+(5-4)}\right]=0.4$.

As $U_{J}\left(\tilde{\mathrm{A}_{1}}\right)=U_{J}\left(\tilde{\mathrm{A}_{2}}\right) \Rightarrow \tilde{\mathrm{A}}_{1} \approx \tilde{\mathrm{A}}_{2}$.

Set 2: $\tilde{\mathrm{A}}_{1}=(3,5,7 ; 1), \tilde{\mathrm{A}}_{2}=\left(4,5, \frac{51}{8} ; 1\right), \tilde{\mathrm{A}}_{3}=(2,3,5 ; 1)$ and $\tilde{\mathrm{A}}_{4}=(6,7,8 ; 1)$.

Here, $x_{\max }=8, x_{\text {min }}=2$.

By using Eq. (1), the following are obtained.

$U_{J}\left(\tilde{\mathrm{A}}_{1}\right)=\frac{1}{2}\left[\frac{(7-2)}{(8-2)-(5-7)}+1-\frac{(8-3)}{(8-2)+(5-3)}\right]=0.5$,

$U_{J}\left(\tilde{\mathrm{A}}_{2}\right)=\frac{1}{2}\left[\frac{\left(\frac{51}{8}-2\right)}{(8-2)-\left(5-\frac{51}{8}\right)}+1-\frac{(8-4)}{(8-2)+(5-4)}\right]=0.5109$.

As $U_{J}\left(\tilde{\mathrm{A}_{1}}\right)<U_{J}\left(\tilde{\mathrm{A}_{2}}\right) \Rightarrow \tilde{\mathrm{A}}_{1} \prec \tilde{\mathrm{A}}_{2}$.

Set 3: $\tilde{\mathrm{A}}_{1}=(3,5,7 ; 1), \tilde{\mathrm{A}}_{2}=\left(4,5, \frac{51}{8} ; 1\right), \tilde{\mathrm{A}}_{3}=(2,3,5 ; 1)$ and $\tilde{\mathrm{A}}_{4}=(10,11,12 ; 1)$.

Here, $x_{\max }=12, x_{\min }=2$.

By using Eq. (1), the following are obtained.

$U_{J}\left(\tilde{\mathrm{A}}_{1}\right)=\frac{1}{2}\left[\frac{(7-2)}{(12-2)-(5-7)}+1-\frac{(12-3)}{(12-2)+(5-3)}\right]=0.3333$, 
$U_{J}\left(\tilde{\mathrm{A}}_{2}\right)=\frac{1}{2}\left[\frac{\left(\frac{51}{8}-2\right)}{(12-2)-\left(5-\frac{51}{8}\right)}+1-\frac{(12-4)}{(12-2)+(5-4)}\right]=0.3287$.

As $U_{J}\left(\tilde{\mathrm{A}_{1}}\right)>U_{J}\left(\tilde{\mathrm{A}_{2}}\right) \Rightarrow \tilde{\mathrm{A}}_{1} \succ \tilde{\mathrm{A}}_{2}$.

From the above three sets, it can be observed that the fuzzy numbers $\tilde{\mathrm{A}}_{1}$ and $\tilde{\mathrm{A}}_{2}$ are identical in all the three sets but, the rankings of $\tilde{\mathrm{A}}_{1}$ and $\tilde{\mathrm{A}}_{2}$ are different. This means that when some new fuzzy numbers are introduced into the given set of fuzzy numbers which change the values of $x_{\max }$ and $x_{\min }$, the ranking method proposed by (Chen, 1985) failed to rank fuzzy numbers.

Example 3.2: (Wang and Luo, 2009) pointed out that when fuzzy numbers have same left, right or total utility values, (Chen's method, 1985) failed to rank them. This can be seen from the following example.

Consider two normal triangular fuzzy numbers $\tilde{\mathrm{A}}_{1}=(3,6,9 ; 1), \tilde{\mathrm{A}}_{2}=(5,6,7 ; 1)$ cited from (Chou et al., 2011). Here, $x_{\max }=12, x_{\min }=2$.

By using Eq. (1), the following are obtained.

$U_{J}\left(\tilde{\mathrm{A}}_{1}\right)=\frac{1}{2}\left[\frac{(9-3)}{(9-3)-(6-9)}+1-\frac{(9-3)}{(9-3)+(6-3)}\right]=0.5$,

$U_{J}\left(\tilde{\mathrm{A}}_{2}\right)=\frac{1}{2}\left[\frac{(7-3)}{(9-3)-(6-7)}+1-\frac{(9-5)}{(9-3)+(6-5)}\right]=0.5$.

As $U_{J}\left(\tilde{\mathrm{A}_{1}}\right)=U_{J}\left(\tilde{\mathrm{A}_{2}}\right) \Rightarrow \tilde{\mathrm{A}_{1}} \approx \tilde{\mathrm{A}}_{2}$.

From the above example it can be concluded that (Chen, 1985) ranking method failed to discriminate fuzzy numbers having same utility values.

\section{Proposed Method}

To address the shortcomings of (Chen, 1985) ranking method, a new revised method of ranking fuzzy numbers based on maximizing and minimizing set on triangular fuzzy numbers formed from generalized trapezoidal fuzzy numbers is presented. In this method, treating a generalized trapezoidal fuzzy number as a trapezoid, the trapezoid is divided into three plane figures namely a triangle, rectangle and a triangle (Fig. 3). The centroids of these plane figures are joined together to form a triangular fuzzy number, and the concept of maximizing set and minimizing set is applied on this fuzzy number. This method uses two left and two right utility values taken along with decision maker's optimism to define the total utility value of each fuzzy number, which serves as a criterion for ranking fuzzy numbers. The revised method can rank fuzzy numbers effectively when a new fuzzy number is added or removed to the set of fuzzy numbers which may change the values of $x_{\max }$. or $x_{\min }$. and even when the total utility values of fuzzy numbers are identical.

Consider $n$ generalized trapezoidal fuzzy numbers $\tilde{\mathrm{A}}_{i}=\left(a_{i}, b_{i}, c_{i}, d_{i} ; w_{i}\right), i=1,2,3, \ldots, n, 0 \leq w_{i} \leq 1$. A triangular fuzzy number (Fig. 3) is formed by treating the trapezoidal fuzzy number $\tilde{\mathrm{A}}_{i}$ as a trapezoid (APQD) and dividing it into three parts, a triangle(APB), rectangle(BPQC) and a triangle(CQD) and 
then by joining their respective centroids $G_{1}=\left(\frac{a_{i}+2 b_{i}}{3}, \frac{w_{i}}{3}\right), G_{2}=\left(\frac{b_{i}+c_{i}}{2}, \frac{w_{i}}{2}\right)$ and $G_{3}=\left(\frac{2 c_{i}+d_{i}}{3}, \frac{w_{i}}{3}\right)$. This is denoted by

$$
\tilde{\mathrm{A}}_{i}^{*}=\left(\frac{a_{i}+2 b_{i}}{3}, \frac{b_{i}+c_{i}}{2}, \frac{2 c_{i}+d_{i}}{3} ; \frac{w_{i}}{2}\right)
$$

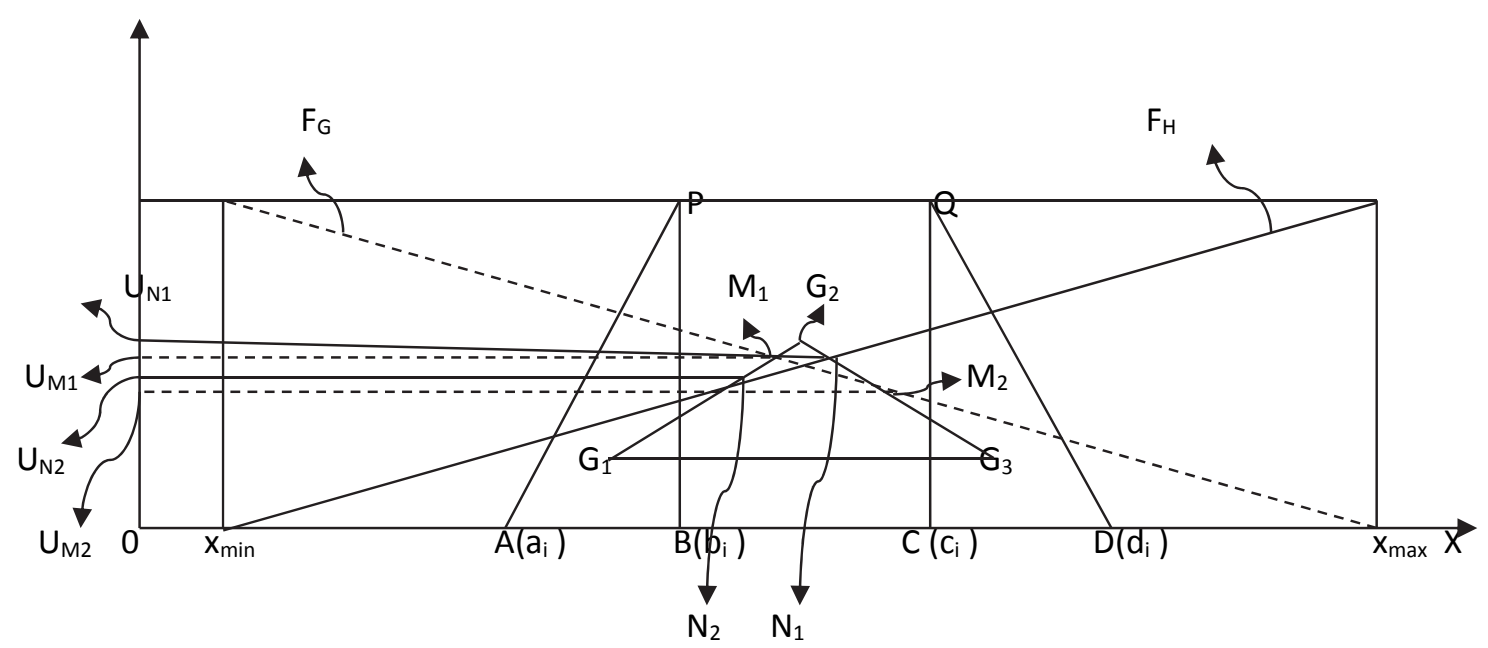

Fig. 3: Maximizing set and minimizing set of fuzzy number

The left membership function of the newly formed triangular fuzzy number $\tilde{\mathrm{A}}_{i}$ is

$$
y=w_{i}\left(\frac{x-a_{i}-b_{i}+c_{i}}{3 c_{i}-2 a_{i}-b_{i}}\right)
$$

The right membership function of the newly formed triangular fuzzy number $\tilde{\mathrm{A}}_{i}$ is

$$
y=w_{i}\left(\frac{x+b_{i}-c_{i}-d_{i}}{3 b_{i}-2 d_{i}-c_{i}}\right)
$$

The membership functions of the newly formed triangular fuzzy number $\tilde{\mathrm{A}}_{i}^{*}$ is

$$
f_{\tilde{\mathrm{A}}_{i}^{*}}(x)=\left\{\begin{array}{lr}
w_{i}\left(\frac{x-a_{i}-b_{i}+c_{i}}{3 c_{i}-2 a_{i}-b_{i}}\right) ; \frac{a_{i}+2 b_{i}}{3} \leq x \leq \frac{b_{i}+c_{i}}{2}, & x=\frac{w_{i}+c_{i}}{2}, \\
w_{i}\left(\frac{x+b_{i}-c_{i}-d_{i}}{3 b_{i}-2 d_{i}-c_{i}}\right) ; & \frac{b_{i}+c_{i}}{2} \leq x \leq \frac{2 c_{i}+d_{i}}{3}, \\
0 ; & \text { otherwise. }
\end{array}\right.
$$

The maximizing set $G$ and the minimizing set $H$ on these triangular fuzzy numbers are: 


$$
\begin{aligned}
& F_{G}(x)=\left\{\begin{array}{lr}
{\left[\frac{w}{2} \frac{\left(x-x_{\min }\right)}{\left(x_{\max }-x_{\min }\right)}\right]^{p} ;} & \text { otherwise. } \\
0 ; & \leq x \leq x_{\max },
\end{array}\right. \\
& F_{H}(x)= \begin{cases}{\left[\frac{w}{2} \frac{\left(x-x_{\max }\right)}{\left(x_{\min }-x_{\max }\right)}\right]^{p} ;} & x_{\min } \leq x \leq x_{\max }, \\
0 ; & \text { otherwise. }\end{cases}
\end{aligned}
$$

Here $x_{\min }=\inf T, x_{\max }=\sup T$ and $T=\bigcup_{i=1}^{n} T_{i}$, where $T_{i}=\left\{x / f_{\tilde{A}_{i}}(x)>0\right\}, w=\inf w_{i}$ and $w_{i}=\sup _{x} f_{\tilde{A}_{i}}(x)$, the constant $p$ varies depending on the application. $p=1$ represents a risk-free membership function, $p=2$ represents a risk-prone membership function and $p=1 / 2$ represents a risk-averse membership function. Throughout this paper, $p=1$ is considered.

In Fig.3, the maximizing set $F_{G}$ intersects the right membership function $f_{\tilde{\mathrm{A}}_{i}^{*}}^{R}(x)$ and the left membership function $f_{\tilde{\mathrm{A}}_{i}^{*}}^{L}(x)$ of the fuzzy number $\tilde{\mathrm{A}}_{i}{ }^{*}$ in points $N_{1}$ and $N_{2}$ whereas the minimizing set $F_{H}$ intersects the left membership function $f_{\tilde{\mathrm{A}}_{i}^{*}}^{L}(x)$ and right membership function $f_{\tilde{\mathrm{A}}_{i}}^{R}(x)$ of the fuzzy number $\tilde{\mathrm{A}}_{i}^{*}$ in points $M_{1}$ and $M_{2}$ respectively.

This method defines two right utility values of each fuzzy number $\tilde{\mathrm{A}}_{i}^{*} ; i=1,2, \ldots, n$ as

$$
\begin{aligned}
& U_{N_{1}}\left(\tilde{\mathrm{A}}_{i}^{*}\right)=\sup _{x}\left(F_{G} \wedge f_{\tilde{\mathrm{A}}_{i}^{*}}^{R}(x)\right) \\
& U_{M_{2}}\left(\tilde{\mathrm{A}}_{i}^{*}\right)=\sup _{x}\left(F_{H} \wedge f_{\tilde{\mathrm{A}}_{i}^{*}}^{R}(x)\right)
\end{aligned}
$$

and two left utility values of each fuzzy number $\tilde{\mathrm{A}}_{i}^{*} ; i=1,2, \ldots, n$ as

$$
\begin{aligned}
& U_{M_{1}}\left(\tilde{\mathrm{A}}_{i}^{*}\right)=\sup _{x}\left(F_{H} \wedge f_{\tilde{\mathrm{A}}_{i}^{*}}^{L}(x)\right) \\
& U_{N_{2}}\left(\tilde{\mathrm{A}}_{i}^{*}\right)=\sup _{x}\left(F_{G} \wedge f_{\tilde{\mathrm{A}}_{i}^{*}}^{L}(x)\right)
\end{aligned}
$$

Therefore,

$$
\begin{aligned}
& U_{N_{1}}\left(\tilde{\mathrm{A}}_{i}^{*}\right)=\frac{w w_{i}\left(x_{\text {min }}+b_{i}-c_{i}-d_{i}\right)}{w\left(3 b_{i}-c_{i}-2 d_{i}\right)-2 w_{i}\left(x_{\max }-x_{\min }\right)} \\
& U_{M_{2}}\left(\tilde{\mathrm{A}}_{i}^{*}\right)=\frac{w w_{i}\left(x_{\text {max }}+b_{i}-c_{i}-d_{i}\right)}{w\left(3 b_{i}-c_{i}-2 d_{i}\right)-2 w_{i}\left(x_{\min }-x_{\max }\right)}
\end{aligned}
$$




$$
\begin{aligned}
& U_{M_{1}}\left(\tilde{\mathrm{A}}_{i}^{*}\right)=\frac{w w_{i}\left(x_{\max }-a_{i}-b_{i}+c_{i}\right)}{w\left(3 c_{i}-2 a_{i}-b_{i}\right)-2 w_{i}\left(x_{\min }-x_{\max }\right)} \\
& U_{N_{2}}\left(\tilde{\mathrm{A}}_{i}^{*}\right)=\frac{w w_{i}\left(x_{\min }-a_{i}-b_{i}+c_{i}\right)}{w\left(3 c_{i}-2 a_{i}-b_{i}\right)-2 w_{i}\left(x_{\max }-x_{\min }\right)}
\end{aligned}
$$

The total utility value of each fuzzy number $\tilde{\mathrm{A}}_{i}$ with index of optimism $\beta$ is defined as

$$
\begin{gathered}
U_{T}^{\beta}\left(\tilde{\mathrm{A}}_{i}^{*}\right)=\frac{1}{2}\left\{\beta\left[U_{N 1}\left(\tilde{\mathrm{A}}_{i}^{*}\right)+\frac{w}{2}-U_{M 2}\left(\tilde{\mathrm{A}}_{i}^{*}\right)\right]+(1-\beta)\left[U_{N 2}\left(\tilde{\mathrm{A}}_{i}^{*}\right)+\frac{w}{2}-U_{M 1}\left(\tilde{\mathrm{A}}_{i}^{*}\right)\right]\right\} \\
U_{T}^{\beta}\left(\tilde{\mathrm{A}}_{i}^{*}\right)=\frac{1}{2}\left\{\begin{array}{c}
\beta\left[\begin{array}{c}
\frac{w w_{i}\left(x_{\min }+b_{i}-c_{i}-d_{i}\right)}{w\left(3 b_{i}-c_{i}-2 d_{i}\right)-2 w_{i}\left(x_{\max }-x_{\min }\right)}+\frac{w}{2} \\
\left.-\frac{w w_{i}\left(x_{\max }+b_{i}-c_{i}-d_{i}\right)}{w\left(3 b_{i}-c_{i}-2 d_{i}\right)-2 w_{i}\left(x_{\min }-x_{\max }\right)}\right]+ \\
(1-\beta)\left[\frac{w w_{i}\left(x_{\min }-a_{i}-b_{i}+c_{i}\right)}{w\left(3 c_{i}-2 a_{i}-b_{i}\right)-2 w_{i}\left(x_{\max }-x_{\min }\right)}\right. \\
+\frac{w}{2}-\frac{w w_{i}\left(x_{\max }-a_{i}-b_{i}+c_{i}\right)}{w\left(3 c_{i}-2 a_{i}-b_{i}\right)-2 w_{i}\left(x_{\min }-x_{\max }\right)}
\end{array}\right]
\end{array}\right\}
\end{gathered}
$$

The index of optimism $\beta$ represents the degree of optimism of a decision maker and larger values of $\beta$ represents a higher degree of optimism. In particular, when $\beta=0, U_{T}^{0}\left(\tilde{\mathrm{A}}_{i}^{*}\right)$ represent a pessimistic decision maker's view point of $\tilde{\mathrm{A}}_{i}^{*}$, conversely, when $\beta=1, U_{T}^{1}\left(\tilde{\mathrm{A}}_{i}^{*}\right)$ represent an optimistic decision maker's view point of $\tilde{\mathrm{A}}_{i}^{*}$. When $\beta=0.5, U_{T}^{1 / 2}\left(\tilde{\mathrm{A}}_{i}^{*}\right)$ represent a moderate decision maker's view point of $\tilde{\mathrm{A}}_{i}^{*}$. The larger the value of $U_{T}^{\beta}\left(\tilde{\mathrm{A}}_{i}^{*}\right)$ is, the higher is the ranking order of the fuzzy number $\tilde{\mathrm{A}}_{i}^{*}$ and hence the fuzzy number $\tilde{\mathrm{A}}_{i}$.

For triangular fuzzy numbers $\tilde{\mathrm{A}}_{i}=\left(a_{i}, b_{i}, d_{i} ; w_{i}\right)$, the newly formed triangular fuzzy numbers are

$$
\tilde{\mathrm{A}}_{i}^{*}=\left(\frac{a_{i}+2 b_{i}}{3}, b_{i}, \frac{2 b_{i}+d_{i}}{3} ; \frac{w_{i}}{2}\right)
$$

and the total utility value of each fuzzy number $\tilde{\mathrm{A}}_{i}^{*} ; i=1,2, \ldots, n$ is given by 


$$
U_{T}^{\beta}\left(\tilde{\mathrm{A}}_{i}^{*}\right)=\frac{1}{2}\left\{\begin{array}{c}
\beta\left[\begin{array}{c}
\frac{w w_{i}\left(x_{\min }-d_{i}\right)}{2\left[w\left(b_{i}-d_{i}\right)-w_{i}\left(x_{\max }-x_{\min }\right)\right]}+\frac{w}{2} \\
-\frac{w w_{i}\left(x_{\max }-d_{i}\right)}{2\left[w\left(b_{i}-d_{i}\right)-w_{i}\left(x_{\min }-x_{\max }\right)\right]}
\end{array}\right]+ \\
(1-\beta)\left[\begin{array}{c}
\frac{w w_{i}\left(x_{\min }-a_{i}\right)}{2\left[w\left(b_{i}-a_{i}\right)-w_{i}\left(x_{\max }-x_{\min }\right)\right]}+\frac{w}{2} \\
-\frac{w w_{i}\left(x_{\max }-a_{i}\right)}{2\left[w\left(b_{i}-a_{i}\right)-w_{i}\left(x_{\min }-x_{\max }\right)\right]}
\end{array}\right]
\end{array}\right\}
$$

\subsection{Numerical Examples}

To demonstrate the new method, the following examples cited from different works are considered.

\section{Example 4.1.1}

Consider two triangular fuzzy numbers $\tilde{\mathrm{A}}_{1}=(3,5,7 ; 1)$ and $\tilde{\mathrm{A}}_{2}=\left(4,5, \frac{51}{8} ; 1\right)$ taken from (Chen, 1985) having same mode and different spreads as shown in Fig. 4.

Here $x_{\max }=7, x_{\min }=3, w=1, w_{1}=w_{2}=1$ and the corresponding triangular fuzzy numbers are: $\tilde{\mathrm{A}}_{1}^{*}=\left(\frac{13}{3}, 5, \frac{17}{3} ; 0.5\right)$ and $\tilde{\mathrm{A}}_{2}^{*}=\left(\frac{14}{3}, 5, \frac{131}{24} ; 0.5\right)$

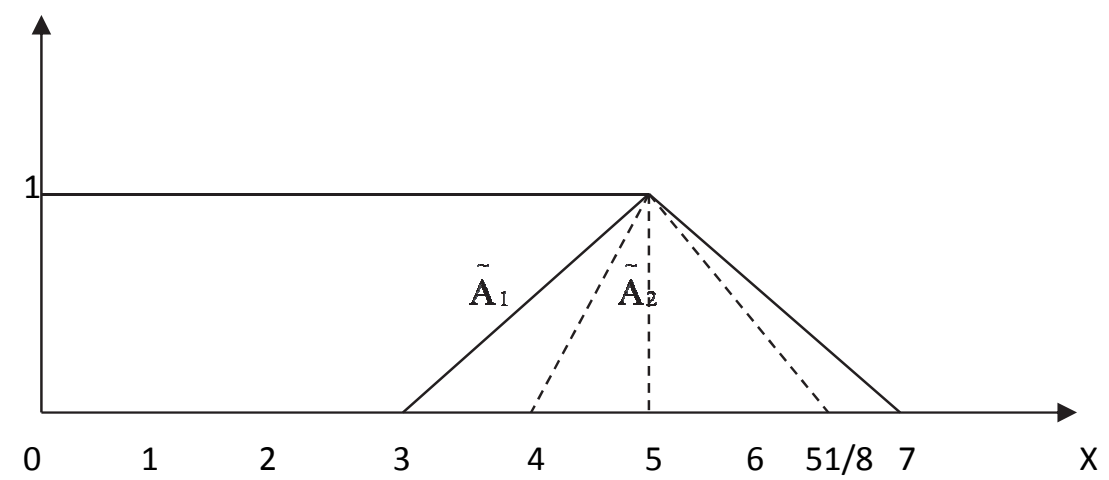

Fig. 4:Diagrammaticrepresentation of fuzzy numbers for Ex. 4.1.1

By using Eq. (19), we get the total utility value of each triangular fuzzy number as:

$U_{T}^{\beta}\left(\tilde{\mathrm{A}}_{2}^{*}\right)=0.1641 \beta+0.1833, U_{T}^{\beta}\left(\tilde{\mathrm{A}}_{1}^{*}\right)=0.3333 \beta+0.0833$

The comparison of fuzzy numbers by decision maker is presented in Table 1.

\section{Table 1}

The comparison of fuzzy numbers by decision maker

\begin{tabular}{|c|c|c|c|}
\hline $\begin{array}{c}\text { Decision maker's } \\
\text { optimism }\end{array}$ & $U_{T}^{\beta}\left(\tilde{\mathrm{A}}_{1}^{*}\right)$ & $U_{T}^{\beta}\left(\tilde{\mathrm{A}}_{2}^{*}\right)$ & Ranking \\
\hline$\beta=0$ & 0.0833 & 0.1833 & $\tilde{\mathrm{A}}_{1}^{*} \prec \tilde{\mathrm{A}}_{2}^{*} \Rightarrow \tilde{\mathrm{A}}_{1} \prec \tilde{\mathrm{A}}_{2}$ \\
\hline$\beta=1$ & 0.4166 & 0.3474 & $\tilde{\mathrm{A}}_{1}^{*} \succ \tilde{\mathrm{A}}_{2}^{*} \Rightarrow \tilde{\mathrm{A}}_{1} \succ \tilde{\mathrm{A}}_{2}$ \\
\hline$\beta=0.5$ & 0.2499 & 0.2653 & $\tilde{\mathrm{A}}_{1}^{*} \prec \tilde{\mathrm{A}}_{2}^{*} \Rightarrow \tilde{\mathrm{A}}_{1} \prec \tilde{\mathrm{A}}_{2}$ \\
\hline
\end{tabular}


From Table 1, it can be seen that a pessimistic decision maker $(\beta=0)$ ranking outcome is $\tilde{\mathrm{A}}_{1} \prec \tilde{\mathrm{A}}_{2}$, an optimistic decision maker $(\beta=1)$ ranking outcome is $\tilde{\mathrm{A}}_{1} \succ \tilde{\mathrm{A}}_{2}$ and a moderate decision maker $(\beta=0.5)$ ranking outcome is $\tilde{\mathrm{A}}_{1} \prec \tilde{\mathrm{A}}_{2}$.

\section{Example 4.1.2}

Consider the following triangular fuzzy numbers $\tilde{\mathrm{A}}_{1}=(3,6,9 ; 1), \tilde{\mathrm{A}}_{2}=(5,6,7 ; 1)$ taken from (Wang and Luo, 2009) having same mode and symmetric spreads as shown in Fig. 5.

Here $x_{\max }=9, x_{\min }=3, w=1, w_{1}=w_{2}=1$ and the corresponding triangular fuzzy numbers are $\tilde{\mathrm{A}}_{1}^{*}=(5,6,7 ; 0.5)$ and $\tilde{\mathrm{A}}_{2}^{*}=\left(\frac{17}{3}, 6, \frac{19}{3} ; 0.5\right)$.

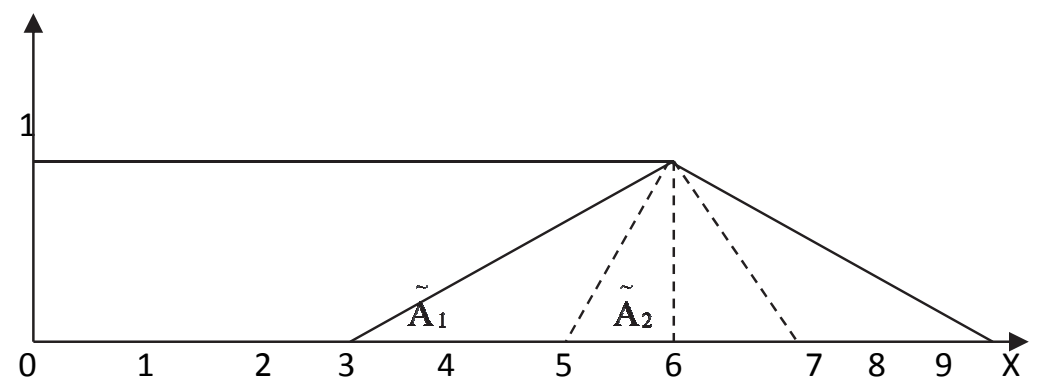

Fig. 5: Diagrammatic representation of fuzzy numbers for Ex. 4.1.2

By using Eq. (19), we get the total utility value of each triangular fuzzy number as:

$U_{T}^{\beta}\left(\tilde{\mathrm{A}}_{1}^{*}\right)=0.3333 \beta+0.0833, U_{T}^{\beta}\left(\tilde{\mathrm{A}}_{2}^{*}\right)=0.0857 \beta+0.2071$

The comparison of fuzzy numbers by decision maker is presented in Table2.

\section{Table 2}

The comparison of fuzzy numbers by decision maker

Decision maker's optimism

\begin{tabular}{|c|c|c|c|}
\hline $\begin{array}{l}\text { sion maker's } \\
\text { optimism }\end{array}$ & $U_{T}^{\beta}\left(\tilde{\mathrm{A}}_{1}^{*}\right)$ & $U_{T}^{\beta}\left(\tilde{\mathrm{A}}_{2}^{*}\right.$ & Ranking \\
\hline$\beta=0$ & 0.0833 & 0.2071 & $\tilde{\mathrm{A}}_{1}^{*} \prec \tilde{\mathrm{A}}_{2}^{*} \Rightarrow \tilde{\mathrm{A}}_{1} \prec \tilde{\mathrm{A}}_{2}$ \\
\hline$\beta=1$ & 0.4166 & 0.2928 & $\tilde{\mathrm{A}}_{1}^{*} \succ \tilde{\mathrm{A}}_{2}^{*} \Rightarrow \tilde{\mathrm{A}}_{1} \succ \tilde{\mathrm{A}}_{2}$ \\
\hline$\beta=0.5$ & 0.2499 & 0.2499 & $\tilde{\mathrm{A}}_{1}^{*} \approx \tilde{\mathrm{A}}_{2}^{*} \Rightarrow \tilde{\mathrm{A}}_{1} \approx \tilde{\mathrm{A}}_{2}$ \\
\hline
\end{tabular}

Ranking

From Table 2, it can be seen that a pessimistic decision maker $(\beta=0)$ the ranking outcome is $\tilde{\mathrm{A}}_{1} \prec \tilde{\mathrm{A}}_{2}$ , an optimistic decision maker $(\beta=1)$ ranking outcome is $\tilde{\mathrm{A}}_{1} \succ \tilde{\mathrm{A}}_{2}$ and a moderate decision maker $(\beta=0.5)$ ranking outcome is $\tilde{\mathrm{A}}_{1} \approx \tilde{\mathrm{A}}_{2}$. 


\section{Example 4.1.3}

Consider a normal and two non-normal fuzzy numbers $\tilde{\mathrm{A}}_{1}=(3,5,7 ; 1), \tilde{\mathrm{A}}_{2}=(3,5,7 ; 0.8)$ and $\tilde{\mathrm{A}}_{3}=(6,7,9,10 ; 0.6)$ taken from (Chen, 1985) as shown in Fig. 6.

Here $x_{\max }=10, x_{\min }=3, w=\inf \{1,0.8,0.6\}=0.6, w_{1}=1, w_{2}=0.8, w_{3}=0.6$ and the corresponding triangular fuzzy numbers are:

$\tilde{\mathrm{A}}_{1}^{*}=\left(\frac{13}{3}, 5, \frac{17}{3} ; 0.5\right) \tilde{\mathrm{A}}_{2}^{*}=\left(\frac{13}{3}, 5, \frac{17}{3} ; 0.4\right) \tilde{\mathrm{A}}_{3}^{*}=\left(\frac{20}{3}, 8, \frac{28}{3} ; 0.3\right)$

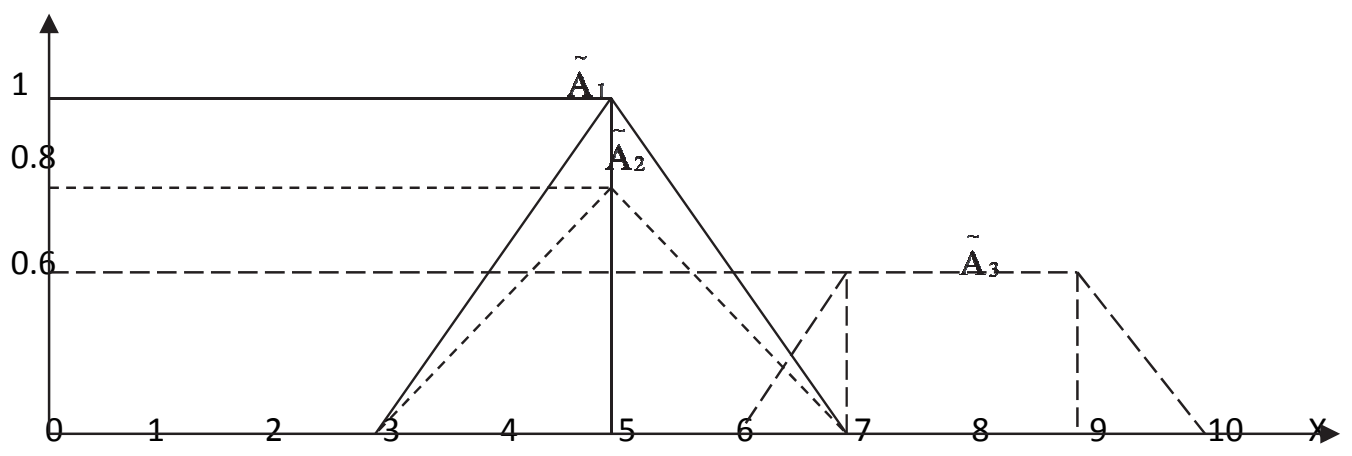

Fig. 6: Diagrammatic representation of fuzzy numbers for Ex. 4.1.3

By using Eq. (19), we get the total utility value of each triangular fuzzy number as:

$$
U_{T}^{\beta}\left(\tilde{\mathrm{A}}_{1}^{*}\right)=0.1236 \beta+0.1219, U_{T}^{\beta}\left(\tilde{\mathrm{A}}_{2}^{*}\right)=0.1122 \beta+0.1265
$$

By using Eq. (17) we get the total utility value of the third triangular fuzzy number as:

$$
U_{T}^{\beta}\left(\tilde{\mathrm{A}}_{3}^{*}\right)=0.2545 \beta+0.2182 \text {. }
$$

The comparison of fuzzy numbers by decision maker is presented in Table 3.

\section{Table 3}

The comparison of fuzzy numbers by decision maker

$\begin{array}{cccc}\begin{array}{c}\text { Decision maker's } \\ \text { optimism }\end{array} & U_{T}^{\beta}\left(\tilde{\mathrm{A}}_{1}^{*}\right) & U_{T}^{\beta}\left(\tilde{\mathrm{A}}_{2}^{*}\right) & U_{T}^{\beta}\left(\tilde{\mathrm{A}}_{3}^{*}\right) \\ & 0.1219 & 0.1265 & 0.2182 \quad \tilde{\mathrm{A}}_{1}^{*} \prec \tilde{\mathrm{A}}_{2}^{*} \prec \tilde{\mathrm{A}}_{3}^{*} \Rightarrow \tilde{\mathrm{A}}_{1} \prec \tilde{\mathrm{A}}_{2} \prec \tilde{\mathrm{A}}_{3} \\ \beta=0 & 0.2455 & 0.2387 & 0.4727 \quad \tilde{\mathrm{A}}_{2}^{*} \prec \tilde{\mathrm{A}}_{1}^{*} \prec \tilde{\mathrm{A}}_{3}^{*} \Rightarrow \tilde{\mathrm{A}}_{2} \prec \tilde{\mathrm{A}}_{1} \prec \tilde{\mathrm{A}}_{3} \\ \beta=1 & 0.1837 & 0.1826 & 0.3454 \quad \tilde{\mathrm{A}}_{2} \prec \tilde{\mathrm{A}}_{1}^{*} \prec \tilde{\mathrm{A}}_{3}^{*} \Rightarrow \tilde{\mathrm{A}}_{2} \prec \tilde{\mathrm{A}}_{1} \prec \tilde{\mathrm{A}}_{3}\end{array}$


From Table 3, it can be seen that a pessimistic decision maker $(\beta=0)$ ranking outcome is $\tilde{\mathrm{A}}_{1} \prec \tilde{\mathrm{A}}_{2} \prec \tilde{\mathrm{A}}_{3}$, an optimistic decision maker $(\beta=1)$ ranking outcome is $\tilde{\mathrm{A}}_{2} \prec \tilde{\mathrm{A}}_{1} \prec \tilde{\mathrm{A}}_{3}$ and a moderate decision maker $(\beta=0.5)$ ranking outcome is $\tilde{\mathrm{A}}_{2} \prec \tilde{\mathrm{A}}_{1} \prec \tilde{\mathrm{A}}_{3}$.

\section{Example 4.1.4}

Let us consider the following sets of fuzzy numbers where $x_{\max }$ or $x_{\min }$ varies.

Set 1: Let $\tilde{\mathrm{A}}_{1}=(3,5,7 ; 1), \tilde{\mathrm{A}}_{2}=\left(4,5, \frac{51}{8} ; 1\right), \tilde{\mathrm{A}}_{3}=(2,3,5 ; 1)$ and $\tilde{\mathrm{A}}_{4}=(8,9,10 ; 1)$ be four triangular fuzzy numbers as shown in Fig. 7.

Here, $x_{\max }=10, x_{\min }=2, w=1, w_{1}=w_{2}=w_{3}=w_{4}=1$ and the corresponding triangular fuzzy numbers are:

$\tilde{\mathrm{A}}_{1}^{*}=\left(\frac{13}{3}, 5, \frac{17}{3} ; 0.5\right), \tilde{\mathrm{A}}_{2}^{*}=\left(\frac{14}{3}, 5, \frac{131}{24} ; 0.5\right), \tilde{\mathrm{A}}_{3}^{*}=\left(\frac{8}{3}, 3, \frac{11}{3} ; 0.5\right)$ and $\tilde{\mathrm{A}}_{4}^{*}=\left(\frac{26}{3}, 9, \frac{28}{3} ; 0.5\right)$.

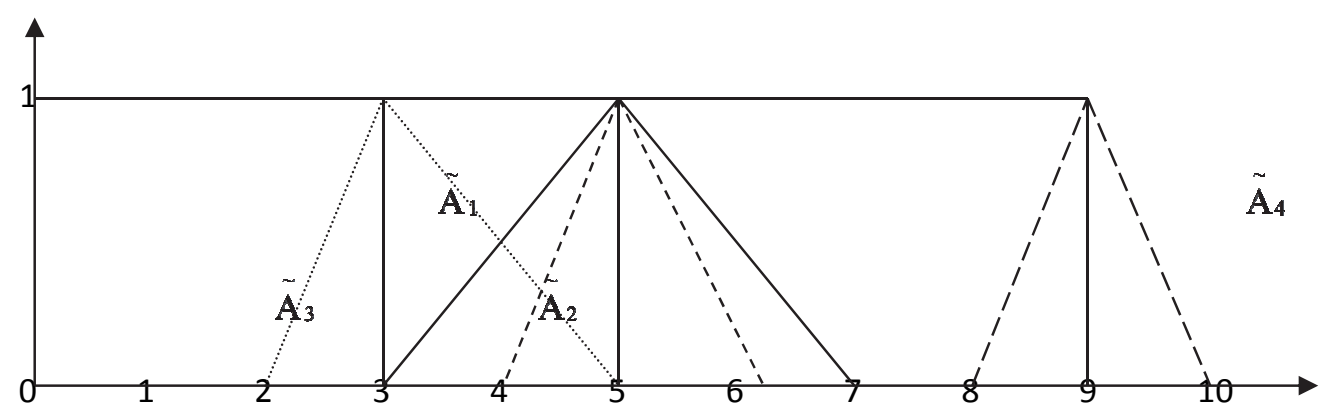

Fig. 7: Diagrammatic representation of fuzzy numbers for Ex. 4.1.4 - Set 1

By using Eq. (19), we get the total utility value of each triangular fuzzy number as:

$U_{T}^{\beta}\left(\tilde{\mathrm{A}}_{1}^{*}\right)=0.15 \beta+0.1, U_{T}^{\beta}\left(\tilde{\mathrm{A}}_{2}^{*}\right)=0.0751 \beta+0.1547$

$U_{T}^{\beta}\left(\tilde{\mathrm{A}}_{3}^{*}\right)=0.0889 \beta+0.0277$ and $U_{T}^{\beta}\left(\tilde{\mathrm{A}}_{4}^{*}\right)=0.0634 \beta+0.4087$

The comparison of fuzzy numbers by decision maker is presented in Table 4.

\section{Table 4}

The comparison of fuzzy numbers by decision maker

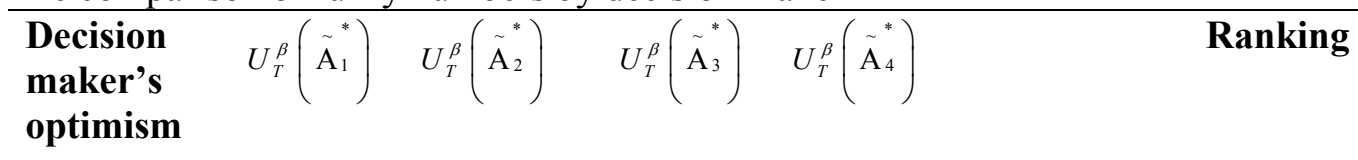

\begin{tabular}{lccccl}
\hline$\beta=0$ & 0.1 & 0.1547 & 0.0277 & 0.8175 & $\tilde{\mathrm{A}}_{3}^{*} \prec \tilde{\mathrm{A}}_{1}^{*} \prec \tilde{\mathrm{A}}_{2}^{*} \prec \tilde{\mathrm{A}}_{4}^{*} \Rightarrow \tilde{\mathrm{A}}_{3} \prec \tilde{\mathrm{A}}_{1} \prec \tilde{\mathrm{A}}_{2} \prec \tilde{\mathrm{A}}_{4}$ \\
$\beta=1$ & 0.25 & 0.2298 & 0.1166 & 0.9444 & $\tilde{\mathrm{A}}_{3}^{*} \prec \tilde{\mathrm{A}}_{1}^{*} \prec \tilde{\mathrm{A}}_{2}^{*} \prec \tilde{\mathrm{A}}_{4}^{*} \Rightarrow \tilde{\mathrm{A}}_{3} \prec \tilde{\mathrm{A}}_{1} \prec \tilde{\mathrm{A}}_{2} \prec \tilde{\mathrm{A}}_{4}$ \\
$\beta=0.5$ & 0.175 & 0.1922 & 0.0721 & 0.8809 & $\tilde{\mathrm{A}}_{3}^{*} \prec \tilde{\mathrm{A}}_{1}^{*} \prec \tilde{\mathrm{A}}_{2}^{*} \prec \tilde{\mathrm{A}}_{4}^{*} \Rightarrow \tilde{\mathrm{A}}_{3} \prec \tilde{\mathrm{A}}_{1} \prec \tilde{\mathrm{A}}_{2} \prec \tilde{\mathrm{A}}_{4}$ \\
& & & & &
\end{tabular}


Set 2: Let $\tilde{\mathrm{A}}_{1}=(3,5,7 ; 1), \tilde{\mathrm{A}}_{2}=\left(4,5, \frac{51}{8} ; 1\right), \tilde{\mathrm{A}}_{3}=(2,3,5 ; 1)$ and $\tilde{\mathrm{A}}_{4}=(6,7,8 ; 1)$ be four triangular fuzzy numbers as shown in Fig. 8.

Here, $x_{\max }=8, x_{\min }=2, w=1, w_{1}=w_{2}=w_{3}=w_{4}=1$ and the corresponding triangular fuzzy numbers are:

$\tilde{\mathrm{A}}_{1}^{*}=\left(\frac{13}{3}, 5, \frac{17}{3} ; 0.5\right), \tilde{\mathrm{A}}_{2}^{*}=\left(\frac{14}{3}, 5, \frac{131}{24} ; 0.5\right), \tilde{\mathrm{A}}_{3}^{*}=\left(\frac{8}{3}, 3, \frac{11}{3} ; 0.5\right)$ and $\tilde{\mathrm{A}}_{4}^{*}=\left(\frac{20}{3}, 7, \frac{22}{3} ; 0.5\right)$.

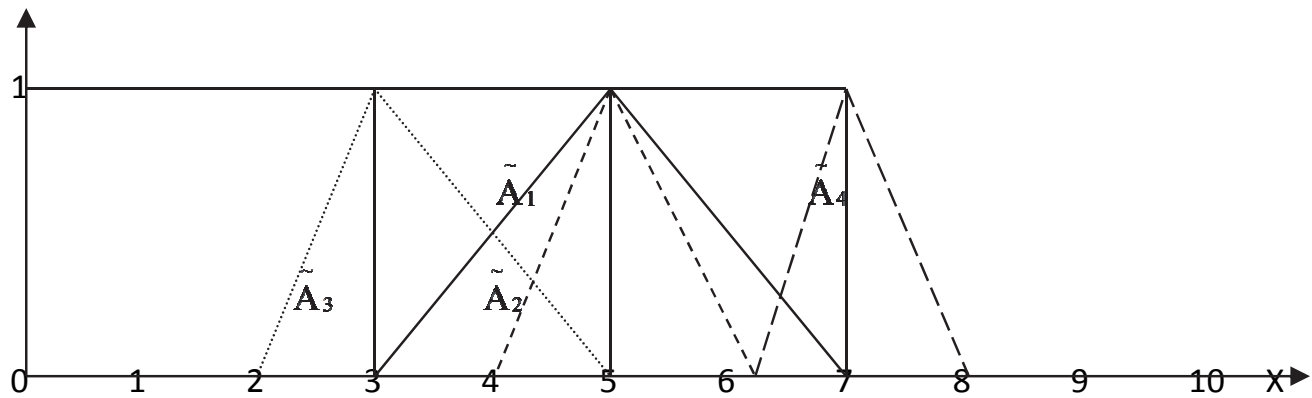

Fig. 8: Diagrammatic representation of fuzzy numbers for Ex. 4.1.4 - Set 2

By using Eq. (19), we get the total utility value of each triangular fuzzy number as:

$U_{T}^{\beta}\left(\tilde{\mathrm{A}}_{1}^{*}\right)=0.18755 \beta+0.1562, U_{T}^{\beta}\left(\tilde{\mathrm{A}}_{2}^{*}\right)=0.1033 \beta+0.2071$

$U_{T}^{\beta}\left(\tilde{\mathrm{A}}_{3}^{*}\right)=0.1205 \beta+0.0351$ and $U_{T}^{\beta}\left(\tilde{\mathrm{A}}_{4}^{*}\right)=0.0857 \beta+0.3786$.

The comparison of fuzzy numbers by decision maker is presented in Table 5 .

Table 5

The comparison of fuzzy numbers by decision maker

\begin{tabular}{|c|c|c|c|c|c|}
\hline $\begin{array}{c}\text { Decision } \\
\text { maker's } \\
\text { optimism } \\
\end{array}$ & $U_{T}^{\beta}\left(\tilde{\mathrm{A}}_{1}^{*}\right)$ & $U_{T}^{\beta}\left(\tilde{\mathrm{A}}_{2}^{*}\right)$ & $U_{T}^{\beta}\left(\tilde{\mathrm{A}}_{3}^{*}\right)$ & $U_{T}^{\beta}\left(\tilde{\mathrm{A}}_{4}^{*}\right)$ & Ranking \\
\hline$\beta=0$ & 0.1562 & 0.2071 & 0.0351 & 0.3786 & $\tilde{\mathrm{A}}_{3}^{*} \prec \tilde{\mathrm{A}}_{1}^{*} \prec \tilde{\mathrm{A}}_{2}^{*} \prec \tilde{\mathrm{A}}_{4}^{*} \Rightarrow \tilde{\mathrm{A}}_{3} \prec \tilde{\mathrm{A}}_{1} \prec \tilde{\mathrm{A}}_{2} \prec \tilde{\mathrm{A}}_{4}$ \\
\hline$\beta=1$ & 0.3437 & 0.3104 & 0.1562 & 0.4643 & $\tilde{\mathrm{A}}_{3}^{*} \prec \tilde{\mathrm{A}}_{1}^{*} \prec \tilde{\mathrm{A}}_{2}^{*} \prec \tilde{\mathrm{A}}_{4}^{*} \Rightarrow \tilde{\mathrm{A}}_{3} \prec \tilde{\mathrm{A}}_{1} \prec \tilde{\mathrm{A}}_{2} \prec \tilde{\mathrm{A}}_{4}$ \\
\hline$\beta=0.5$ & 0.2499 & 0.2587 & 0.0953 & 0.4214 & $\tilde{\mathrm{A}}_{3}^{*} \prec \tilde{\mathrm{A}}_{1}^{*} \prec \tilde{\mathrm{A}}_{2}^{*} \prec \tilde{\mathrm{A}}_{4}^{*} \Rightarrow \tilde{\mathrm{A}}_{3} \prec \tilde{\mathrm{A}}_{1} \prec \tilde{\mathrm{A}}_{2} \prec \tilde{\mathrm{A}}_{4}$ \\
\hline
\end{tabular}

Set 3: Let $\tilde{\mathrm{A}}_{1}=(3,5,7 ; 1), \tilde{\mathrm{A}}_{2}=\left(4,5, \frac{51}{8} ; 1\right), \tilde{\mathrm{A}}_{3}=(2,3,5 ; 1)$ and $\tilde{\mathrm{A}}_{4}=(10,11,12 ; 1)$ be four triangular fuzzy numbers as shown in Fig. 9.

Here, $x_{\max }=8, x_{\min }=2, w=1, w_{1}=w_{2}=w_{3}=w_{4}=1$ and the corresponding triangular fuzzy numbers are:

$$
\tilde{\mathrm{A}}_{1}^{*}=\left(\frac{13}{3}, 5, \frac{17}{3} ; 0.5\right), \tilde{\mathrm{A}}_{2}^{*}=\left(\frac{14}{3}, 5, \frac{131}{24} ; 0.5\right), \tilde{\mathrm{A}}_{3}^{*}=\left(\frac{8}{3}, 3, \frac{11}{3} ; 0.5\right) \text { and } \tilde{\mathrm{A}}_{4}^{*}=\left(\frac{32}{3}, 11, \frac{34}{3} ; 0.5\right)
$$




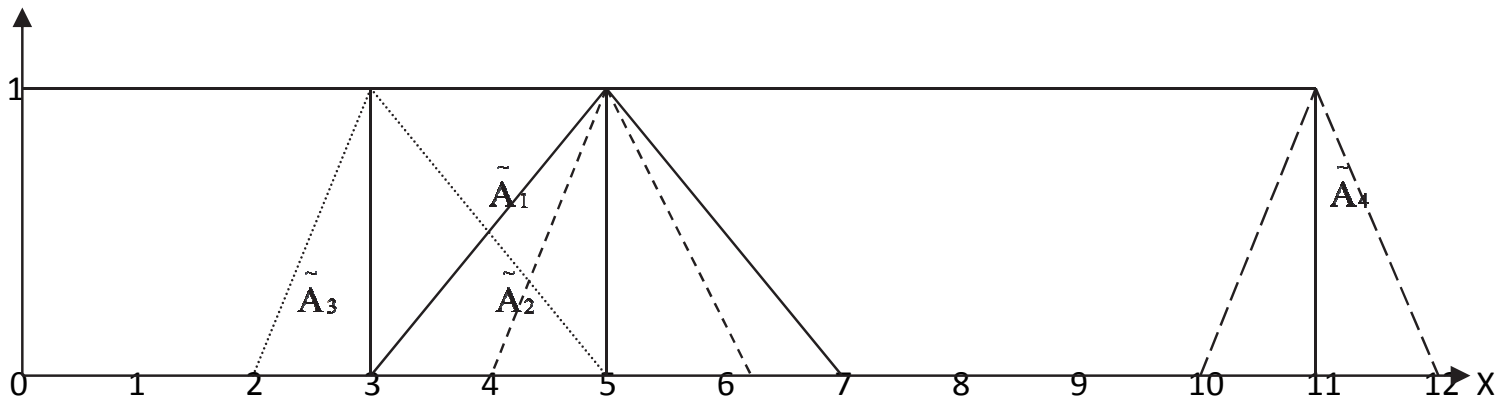

Fig. 9: Diagrammatic representation of fuzzy numbers for Ex. 4.1.4 - Set 3

$U_{T}^{\beta}\left(\mathrm{A}_{3}\right)=0.071 \beta+0.0227 U_{T}^{\beta}\left(\mathrm{A}_{4}\right)=0.0505 \beta+0.4267$

The comparison of fuzzy numbers by decision makers is presented in Table 6 .

Table 6

The comparison of fuzzy numbers by decision makers

\begin{tabular}{|c|c|c|c|c|c|}
\hline $\begin{array}{c}\text { Decision } \\
\text { maker's } \\
\text { optimism }\end{array}$ & $U_{T}^{\beta}\left(\tilde{\mathrm{A}}_{1}^{*}\right)$ & $U_{T}^{\beta}\left(\tilde{\mathrm{A}}_{2}^{*}\right)$ & $U_{T}^{\beta}\left(\tilde{\mathrm{A}}_{3}^{*}\right)$ & $U_{T}^{\beta}\left(\tilde{\mathrm{A}}_{4}^{*}\right)$ & Ranking \\
\hline$\beta=0$ & 0.0937 & 0.1237 & 0.0227 & 0.4267 & $\tilde{\mathrm{A}}_{3}^{*} \prec \tilde{\mathrm{A}}_{1}^{*} \prec \tilde{\mathrm{A}}_{2}^{*} \prec \tilde{\mathrm{A}}_{4}^{*} \Rightarrow \tilde{\mathrm{A}}_{3} \prec \tilde{\mathrm{A}}_{1} \prec \tilde{\mathrm{A}}_{2} \prec \tilde{\mathrm{A}}_{4}$ \\
\hline$\beta=1$ & 0.1978 & 0.183 & 0.0937 & 0.4772 & $\tilde{\mathrm{A}}_{3}^{*} \prec \tilde{\mathrm{A}}_{1}^{*} \prec \tilde{\mathrm{A}}_{2}^{*} \prec \tilde{\mathrm{A}}_{4}^{*} \Rightarrow \tilde{\mathrm{A}}_{3} \prec \tilde{\mathrm{A}}_{1} \prec \tilde{\mathrm{A}}_{2} \prec \tilde{\mathrm{A}}_{4}$ \\
\hline$\beta=0.5$ & 0.1457 & 0.1533 & 0.0582 & 0.4519 & $\tilde{\mathrm{A}}_{3}^{*} \prec \tilde{\mathrm{A}}_{1}^{*} \prec \tilde{\mathrm{A}}_{2}^{*} \prec \tilde{\mathrm{A}}_{4}^{*} \Rightarrow \tilde{\mathrm{A}}_{3} \prec \tilde{\mathrm{A}}_{1} \prec \tilde{\mathrm{A}}_{2} \prec \tilde{\mathrm{A}}_{4}$ \\
\hline
\end{tabular}

From Tables 4, 5 and 6 it can be concluded that the shortcomings of the (Chen, 1985) method have been overcome by the new method. Throughout the Sets 1, 2 and 3 a pessimistic decision maker $(\beta=0)$, an optimistic decision maker $(\beta=1)$ and a moderate decision maker $(\beta=0.5)$ ranks the fuzzy numbers $\tilde{\mathrm{A}}_{1}, \tilde{\mathrm{A}}_{2}, \tilde{\mathrm{A}}_{3}$ and $\tilde{\mathrm{A}}_{4}$ as $\tilde{\mathrm{A}}_{3} \prec \tilde{\mathrm{A}}_{1} \prec \tilde{\mathrm{A}}_{2} \prec \tilde{\mathrm{A}}_{4}$. The results of this example show that when $x_{\text {max }}$. or $x_{\min }$.are varied, the ranking outcome of the fuzzy numbers do not alter.

\section{Example 4.1.5}

Consider two trapezoidal fuzzy numbers $\mathrm{A}_{1}=(0,0.4,0.6,0.8 ; 1)$ and $\mathrm{A}_{2}=(0.1,0.6,0.7,0.8 ; 1)$ with zero inside the support cited from (Chen and Chen, 2007)

Here $x_{\text {max. }}=0.8$ and $x_{\text {min. }}=0, w=1, w_{1}=w_{2}=1$ and the corresponding triangular fuzzy numbers are: $\tilde{\mathrm{A}}_{1}^{*}=\left(\frac{0.8}{3}, 0.5, \frac{2}{3} ; 0.5\right), \tilde{\mathrm{A}}_{2}^{*}=\left(\frac{1.3}{3}, 0.65, \frac{1.1}{3} ; 0.5\right)$.

By using Eq. (17), we get the total utility value of each triangular fuzzy number as:

$U_{T}^{\beta}\left(\tilde{\mathrm{A}}_{1}^{*}\right)=-0.4286+0.7458 \beta, U_{T}^{\beta}\left(\tilde{\mathrm{A}}_{2}^{*}\right)=0.1121+0.3977 \beta$.

The comparison of fuzzy numbers by decision makers is presented in Table 7. 
Table 7

The comparison of fuzzy numbers by decision makers

\begin{tabular}{|c|c|c|c|}
\hline $\begin{array}{c}\text { Decision } \\
\text { maker's } \\
\text { optimism }\end{array}$ & $U_{T}^{\beta}\left(\tilde{\mathrm{A}}_{1}^{*}\right)$ & $U_{T}^{\beta}\left(\tilde{\mathrm{A}}_{2}^{*}\right)$ & Ranking \\
\hline$\beta=0$ & -0.4286 & 0.1121 & $\tilde{\mathrm{A}}_{1}^{*} \prec \tilde{\mathrm{A}}_{2}^{*} \Rightarrow \tilde{\mathrm{A}}_{1} \prec \tilde{\mathrm{A}}_{2}$ \\
\hline$\beta=1$ & 0.3172 & 0.5098 & $\tilde{\mathrm{A}}_{1}^{*} \prec \tilde{\mathrm{A}}_{2}^{*} \Rightarrow \tilde{\mathrm{A}}_{1} \prec \tilde{\mathrm{A}}_{2}$ \\
\hline$\beta=0.5$ & -0.0557 & 0.3109 & $\tilde{\mathrm{A}}_{1}^{*} \prec \tilde{\mathrm{A}}_{2}^{*} \Rightarrow \tilde{\mathrm{A}}_{1} \prec \tilde{\mathrm{A}}_{2}$ \\
\hline
\end{tabular}

From Table 7 it can be concluded that a pessimistic decision maker $(\beta=0)$, an optimistic decision maker $(\beta=1)$ and a moderate decision maker $(\beta=0.5)$ ranks the fuzzy numbers $\tilde{\mathrm{A}}_{1}$ and $\tilde{\mathrm{A}}_{2}$ as $\tilde{A_{1}} \prec \tilde{A}_{2}$. These results are in coincidence with that of (Yager, 1981) and (Yao and Wu, 2000).

\section{Example 4.1.6}

Consider the fuzzy numbers $\mathrm{A}_{1}=(-0.5,-0.3,-0.1 ; 1)$ and $\mathrm{A}_{2}=(0.1,0.3,0.5 ; 1)$ with negative support cited from (Lee and Chen, 2008).

Here $x_{\max }=0.5$ and $x_{\text {min. }}=0.1, w=1, w_{1}=w_{2}=1$ and the corresponding triangular fuzzy numbers are:

$\tilde{\mathrm{A}}_{1}^{*}=\left(\frac{-1.1}{3},-0.3, \frac{-0.7}{3} ; 0.5\right), \tilde{\mathrm{A}}_{2}^{*}=\left(\frac{0.7}{3}, 0.3, \frac{1.1}{3} ; 0.5\right)$.

By using Eq. (19), we get the total utility value of each triangular fuzzy number as:

$U_{T}^{\beta}\left(\tilde{\mathrm{A}}_{1}^{*}\right)=-0.9167+0.6667 \beta, U_{T}^{\beta}\left(\tilde{\mathrm{A}}_{2}^{*}\right)=0.0833+0.6667 \beta$.

The comparison of fuzzy numbers by decision makers is presented in Table 8 .

Table 8

The comparison of fuzzy numbers by decision makers

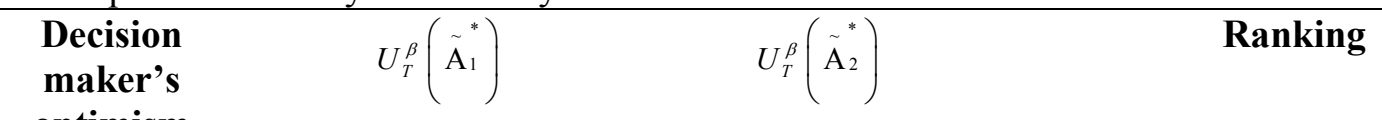

\section{optimism}

\begin{tabular}{lccl}
\hline$\beta=0$ & -0.9167 & 0.0833 & $\tilde{\mathrm{A}}_{1}^{*} \prec \tilde{\mathrm{A}}_{2}^{*} \Rightarrow \tilde{\mathrm{A}}_{1} \prec \tilde{\mathrm{A}}_{2}$ \\
$\beta=1$ & -0.25 & 0.75 & $\tilde{\mathrm{A}}_{1}^{*} \prec \tilde{\mathrm{A}}_{2}^{*} \Rightarrow \tilde{\mathrm{A}}_{1} \prec \tilde{\mathrm{A}}_{2}$ \\
$\beta=0.5$ & -0.5833 & 0.4166 & $\tilde{\mathrm{A}}_{1}^{*} \prec \tilde{\mathrm{A}}_{2}^{*} \Rightarrow \tilde{\mathrm{A}}_{1} \prec \tilde{\mathrm{A}}_{2}$ \\
\hline
\end{tabular}

From Table 8 it can be concluded that a pessimistic decision maker $(\beta=0)$, an optimistic decision maker $(\beta=1)$ and a moderate decision maker $(\beta=0.5)$ ranks the fuzzy numbers $\tilde{\mathrm{A}}_{1}$ and $\tilde{\mathrm{A}}_{2}$ as $\tilde{A_{1}} \prec \tilde{A_{2}}$. These results are in coincidence with that of (Yager, 1981) and (Yao and Wu, 2000). 
As $-\tilde{\mathrm{A}_{1}}=(0.1,0.3,0.5 ; 1)=\tilde{\mathrm{A}_{2}}$ and $-\tilde{\mathrm{A}_{2}}=(-0.5,-0.3,-0.1 ; 1)=\tilde{\mathrm{A}_{1}}$, we can conclude that if $\tilde{\mathrm{A}_{1}} \prec \tilde{\mathrm{A}_{2}}$ then $-\tilde{\mathrm{A}_{2}} \prec-\tilde{\mathrm{A}_{1}}$.

\section{Comparative study}

In this section, the new method is compared with four sets of fuzzy numbers taken from Yao and $\mathrm{Wu}(2000)$. The ranking outcomes are compared with few existing methods in literature like (Yager, 1981; Chen, 1985; Chu \&Tsao, 2002; Fortemps\&Roubens, 1996; Yao \& Wu, 2000; Chen \& Chen, 2009; Abbasbandy\&Asady, 2006). A comparative statement showing the ranking outcomes of various ranking methods and the new method is presented in Table 9.

Set 1: $\tilde{\mathrm{A}}_{1}=(0.4,0.5,1 ; 1), \tilde{\mathrm{A}}_{2}=(0.4,0.7,1 ; 1)$ and $\tilde{\mathrm{A}}_{3}=(0.4,0.9,1 ; 1)$

Here, $x_{\max }=1, x_{\min }=0.4, w=1, w_{1}=w_{2}=w_{3}=1$ and the corresponding fuzzy numbers are $\tilde{\mathrm{A}}_{1}^{*}=\left(\frac{1.4}{3}, 0.5, \frac{2}{3} ; 0.5\right), \tilde{\mathrm{A}}_{2}^{*}=\left(\frac{1.8}{3}, 0.7, \frac{2.4}{3} ; 0.5\right), \tilde{\mathrm{A}}_{3}^{*}=\left(\frac{2.2}{3}, 0.9, \frac{2.8}{3} ; 0.5\right)$.

By using Eq. (19), we get the total utility value of each triangular fuzzy number as: $U_{T}^{\beta}\left(\tilde{\mathrm{A}}_{1}^{*}\right)=0.0357+0.3506 \beta, U_{T}^{\beta}\left(\tilde{\mathrm{A}}_{2}^{*}\right)=0.0833+0.3333 \beta, U_{T}^{\beta}\left(\tilde{\mathrm{A}}_{3}^{*}\right)=0.1136+0.3506 \beta$

Set 2: $\tilde{\mathrm{A}}_{1}=(0.3,0.4,0.7,0.9 ; 1), \tilde{\mathrm{A}}_{2}=(0.3,0.7,0.9 ; 1)$ and $\tilde{\mathrm{A}}_{3}=(0.5,0.7,0.9 ; 1)$

Here, $\quad x_{\max }=0.9, x_{\min }=0.3, w=1, w_{1}=w_{2}=w_{3}=1$ and the corresponding fuzzy numbers are $\tilde{\mathrm{A}}_{1}^{*}=\left(\frac{1.1}{3}, \frac{1.1}{2}, \frac{2.3}{3} ; 0.5\right), \tilde{\mathrm{A}}_{2}^{*}=\left(\frac{1.7}{3}, 0.7, \frac{2.3}{3} ; 0.5\right), \tilde{\mathrm{A}}_{3}^{*}=\left(\frac{1.9}{3}, 0.7, \frac{2.3}{3} ; 0.5\right)$.

By using Eq. (17) and Eq. (19), we get the total utility value of each triangular fuzzy number as: $U_{T}^{\beta}\left(\tilde{\mathrm{A}}_{1}^{*}\right)=-1.0543-0.0156 \beta, U_{T}^{\beta}\left(\tilde{\mathrm{A}}_{2}^{*}\right)=0.1+0.3375 \beta$ and $U_{T}^{\beta}\left(\tilde{\mathrm{A}}_{3}^{*}\right)=0.25+0.1875 \beta$

Set 3: $\tilde{\mathrm{A}}_{1}=(0.3,0.5,0.7 ; 1), \tilde{\mathrm{A}}_{2}=(0.3,0.5,0.8,0.9 ; 1)$ and $\tilde{\mathrm{A}}_{3}=(0.3,0.5,0.9 ; 1)$

Here, $\quad x_{\max }=0.9, x_{\min }=0.3, w=1, w_{1}=w_{2}=w_{3}=1$ and the corresponding fuzzy numbers are $\tilde{\mathrm{A}}_{1}^{*}=\left(\frac{1.3}{3}, 0.5, \frac{1.7}{3} ; 0.5\right), \tilde{\mathrm{A}}_{2}^{*}=\left(\frac{1.3}{3}, \frac{1.3}{2}, \frac{2.5}{3} ; 0.5\right), \tilde{\mathrm{A}}_{3}^{*}=\left(\frac{1.3}{3}, 0.5, \frac{1.9}{3} ; 0.5\right)$.

By using Eq. (17) and Eq. (19), we get the total utility value of each triangular fuzzy number as: $U_{T}^{\beta}\left(\tilde{\mathrm{A}}_{1}^{*}\right)=0.0625+0.1875 \beta, U_{T}^{\beta}\left(\tilde{\mathrm{A}}_{2}^{*}\right)=1.57-2.6243 \beta$ and $U_{T}^{\beta}\left(\tilde{\mathrm{A}}_{3}^{*}\right)=0.0625+0.3375 \beta$.

Set 4: $\tilde{\mathrm{A}}_{1}=(0.0,0.4,0.7,0.8 ; 1), \tilde{\mathrm{A}_{2}}=(0.2,0.5,0.9 ; 1)$ and $\tilde{\mathrm{A}_{3}}=(0.1,0.6,0.8 ; 1)$

Here, $\quad x_{\max }=0.9, x_{\min }=0.0, w=1, w_{1}=w_{2}=w_{3}=1$ and the corresponding fuzzy numbers are $\tilde{\mathrm{A}}_{1}^{*}=\left(\frac{0.8}{3}, \frac{1.1}{2}, \frac{2.2}{3} ; 0.5\right), \tilde{\mathrm{A}}_{2}^{*}=\left(\frac{1.2}{3}, 0.5, \frac{1.9}{3} ; 0.5\right), \tilde{\mathrm{A}}_{3}^{*}=\left(\frac{1.3}{3}, 0.6, \frac{2}{3} ; 0.5\right)$.

By using Eq. (17) and Eq. (19), we get the total utility value of each triangular fuzzy number as: $U_{T}^{\beta}\left(\tilde{\mathrm{A}}_{1}^{*}\right)=1.6753 \beta-1.3785, U_{T}^{\beta}\left(\tilde{\mathrm{A}}_{2}^{*}\right)=0.4980 \beta+0.375$ and $U_{T}^{\beta}\left(\tilde{\mathrm{A}}_{3}^{*}\right)=0.3872 \beta+0.3303$ 
Table 9

The comparison of different methods

\begin{tabular}{|c|c|c|c|c|}
\hline Methods & Set 1 & Set 2 & Set 3 & Set 4 \\
\hline (Yager, 1981) & $\tilde{\mathrm{A}}_{1} \prec \tilde{\mathrm{A}}_{2} \prec \tilde{\mathrm{A}}_{3}$ & $\tilde{\mathrm{A}}_{1} \prec \tilde{\mathrm{A}}_{2} \prec \tilde{\mathrm{A}}_{3}$ & $\tilde{\mathrm{A}}_{1} \prec \tilde{\mathrm{A}}_{3} \prec \tilde{\mathrm{A}}_{2}$ & $\tilde{\mathrm{A}}_{1} \prec \tilde{\mathrm{A}}_{2} \approx \tilde{\mathrm{A}}_{3}$ \\
\hline (Chen, 1985) & $\tilde{\mathrm{A}}_{1} \prec \tilde{\mathrm{A}}_{2} \prec \tilde{\mathrm{A}}_{3}$ & $\tilde{\mathrm{A}}_{1} \prec \tilde{\mathrm{A}}_{2} \prec \tilde{\mathrm{A}}_{3}$ & $\tilde{\mathrm{A}}_{1} \prec \tilde{\mathrm{A}}_{3} \prec \tilde{\mathrm{A}}_{2}$ & $\tilde{\mathrm{A}}_{1} \prec \tilde{\mathrm{A}}_{2} \prec \tilde{\mathrm{A}}_{3}$ \\
\hline (Chu and Tsao, 2002) & $\tilde{\mathrm{A}}_{1} \prec \tilde{\mathrm{A}}_{2} \prec \tilde{\mathrm{A}}_{3}$ & $\tilde{\mathrm{A}}_{1} \prec \tilde{\mathrm{A}}_{2} \prec \tilde{\mathrm{A}}_{3}$ & $\tilde{\mathrm{A}}_{1} \prec \tilde{\mathrm{A}}_{3} \prec \tilde{\mathrm{A}}_{2}$ & $\tilde{\mathrm{A}}_{1} \prec \tilde{\mathrm{A}}_{2} \prec \tilde{\mathrm{A}}_{3}$ \\
\hline $\begin{array}{l}\text { (Fortemps and Roubens, } \\
\text { 1996) }\end{array}$ & $\tilde{\mathrm{A}}_{1} \prec \tilde{\mathrm{A}}_{2} \prec \tilde{\mathrm{A}}_{3}$ & $\tilde{\mathrm{A}}_{1} \prec \tilde{\mathrm{A}}_{2} \prec \tilde{\mathrm{A}}_{3}$ & $\tilde{\mathrm{A}}_{1} \prec \tilde{\mathrm{A}}_{3} \prec \tilde{\mathrm{A}}_{2}$ & $\mathrm{~A}_{1} \prec \mathrm{A}_{2} \approx \mathrm{A}_{3}$ \\
\hline (Yao and $\mathrm{Wu}, 2000$ ) & $\tilde{\mathrm{A}}_{1} \prec \tilde{\mathrm{A}}_{2} \prec \tilde{\mathrm{A}}_{3}$ & $\tilde{\mathrm{A}}_{1} \prec \tilde{\mathrm{A}}_{2} \prec \tilde{\mathrm{A}}_{3}$ & $\tilde{\mathrm{A}}_{1} \prec \tilde{\mathrm{A}}_{3} \prec \tilde{\mathrm{A}}_{2}$ & $\tilde{\mathrm{A}}_{1} \prec \tilde{\mathrm{A}}_{2} \approx \tilde{\mathrm{A}}_{3}$ \\
\hline $\begin{array}{l}\text { (Abbasbandy and Asady, } \\
\text { 2006) }\end{array}$ & $\tilde{\mathrm{A}}_{1} \prec \tilde{\mathrm{A}}_{2} \prec \tilde{\mathrm{A}}_{3}$ & $\tilde{\mathrm{A}}_{1} \prec \tilde{\mathrm{A}}_{2} \prec \tilde{\mathrm{A}}_{3}$ & $\tilde{\mathrm{A}}_{1} \prec \tilde{\mathrm{A}}_{3} \prec \tilde{\mathrm{A}}_{2}$ & $\tilde{\mathrm{A}}_{1} \prec \tilde{\mathrm{A}}_{2} \approx \tilde{\mathrm{A}}_{3}$ \\
\hline (Chen and Chen, 2009) & $\tilde{\mathrm{A}}_{1} \prec \tilde{\mathrm{A}}_{2} \prec \tilde{\mathrm{A}}_{3}$ & $\tilde{\mathrm{A}}_{1} \prec \tilde{\mathrm{A}}_{2} \prec \tilde{\mathrm{A}}_{3}$ & $\tilde{\mathrm{A}}_{1} \prec \tilde{\mathrm{A}}_{3} \prec \tilde{\mathrm{A}}_{2}$ & $\tilde{\mathrm{A}}_{1} \prec \tilde{\mathrm{A}}_{3} \prec \tilde{\mathrm{A}}_{2}$ \\
\hline \multicolumn{5}{|l|}{ New Method } \\
\hline$\beta=0$ & $\tilde{\mathrm{A}}_{1} \prec \tilde{\mathrm{A}}_{2} \prec \tilde{\mathrm{A}}_{3}$ & $\tilde{\mathrm{A}}_{1} \prec \tilde{\mathrm{A}}_{2} \prec \tilde{\mathrm{A}}_{3}$ & $\tilde{\mathrm{A}}_{1} \approx \tilde{\mathrm{A}}_{3} \prec \tilde{\mathrm{A}}_{2}$ & $\tilde{\mathrm{A}}_{1} \prec \tilde{\mathrm{A}}_{3} \prec \tilde{\mathrm{A}}_{2}$ \\
\hline$\beta=1$ & $\tilde{\mathrm{A}}_{1} \prec \tilde{\mathrm{A}}_{2} \prec \tilde{\mathrm{A}}_{3}$ & $\tilde{\mathrm{A}}_{1} \prec \tilde{\mathrm{A}}_{2} \approx \tilde{\mathrm{A}}_{3}$ & $\tilde{\mathrm{A}}_{2} \prec \tilde{\mathrm{A}}_{1} \prec \tilde{\mathrm{A}}_{3}$ & $\tilde{\mathrm{A}}_{1} \prec \tilde{\mathrm{A}}_{3} \prec \tilde{\mathrm{A}}_{2}$ \\
\hline$\beta=0.5$ & $\tilde{\mathrm{A}}_{1} \prec \tilde{\mathrm{A}}_{2} \prec \tilde{\mathrm{A}}_{3}$ & $\tilde{\mathrm{A}}_{1} \prec \tilde{\mathrm{A}}_{2} \prec \tilde{\mathrm{A}}_{3}$ & $\tilde{\mathrm{A}}_{1} \prec \tilde{\mathrm{A}}_{3} \prec \tilde{\mathrm{A}}_{2}$ & $\tilde{\mathrm{A}}_{1} \prec \tilde{\mathrm{A}}_{3} \prec \tilde{\mathrm{A}}_{2}$ \\
\hline
\end{tabular}

\section{Conclusions}

This paper proposes a new method on ranking fuzzy numbers which improves (Chen's, 1985) ranking method. This method considers the triangular fuzzy numbers formed by centroids obtained by splitting generalized trapezoidal fuzzy numbers into three parts and finds two left and two right utility values of the triangular fuzzy numbers clubbed with the decision maker's optimistic attitude to define a total utility value which serves as a criterion for ranking fuzzy numbers. This method is more consistent with human intuition than (Chen's, 1985) ranking method and can efficiently rank various types of fuzzy numbers like normal, non-normal triangular and trapezoidal fuzzy numbers. This method can rank fuzzy numbers effectively when $x_{\max }$. or $x_{\min }$. is changed and it can also rank fuzzy numbers having same left, right or total utility values in an effective manner.

\section{Conflict of interest}

The author declares that there is no conflict of interest regarding the publication of this paper.

\section{References}

Abbasbandy, S., \&Asady, B. (2006). Ranking of fuzzy numbers by sign distance. Information Sciences, 176(16), 2405-2416.

Chen, S. H. (1985). Ranking fuzzy numbers with maximizing set and minimizing set. Fuzzy sets and Systems, 17(2), 113-129.

Chen, S. M., \& Chen, J. H. (2009). Fuzzy risk analysis based on ranking generalized fuzzy numbers with different heights and different spreads. Expert systems with applications, 36(3), 6833-6842. 
Chen, S. J., \& Chen, S. M. (2007). Fuzzy risk analysis based on the ranking of generalized trapezoidal fuzzy numbers. Applied intelligence, 26(1), 1-11.

Cheng, C. H. (1998). A new approach for ranking fuzzy numbers by distance method. Fuzzy sets and systems, 95(3), 307-317.

Chu, T. C., \&Tsao, C. T. (2002). Ranking fuzzy numbers with an area between the centroid point and original point. Computers \& Mathematics with Applications, 43(1-2), 111-117.

Chou, S. Y., Dat, L. Q., \& Vincent, F. Y. (2011). A revised method for ranking fuzzy numbers using maximizing set and minimizing set. Computers \& Industrial Engineering, 61(4), 1342-1348.

Dubois, D., \&Prade, H. (1978). Operations on fuzzy numbers. International Journal of systems science, 9(6), 613-626.

Fortemps, P., \&Roubens, M. (1996). Ranking and defuzzification methods based on area compensation. Fuzzy Sets and Systems, 82(3), 319-330.

Jain, R. (1978). Decision-making in the presence of a fuzzy variable. IEEE Transactions on Systems, Man and Cybernetics, 6, 698-703.

Lee, L. W., \& Chen, S. M. (2008). Fuzzy risk analysis based on fuzzy numbers with different shapes and different deviations. Expert Systems with Applications, 34(4), 2763-2771.

Wang, Y. M., \& Luo, Y. (2009). Area ranking of fuzzy numbers based on positive and negative ideal points. Computers \& Mathematics with Applications, 58(9), 1769-1779.

Yager, R. R. (1981). A procedure for ordering fuzzy subsets of the unit interval. Information sciences, 24(2), 143-161.

Yao, J. S., \& Wu, K. (2000). Ranking fuzzy numbers based on decomposition principle and signed distance. Fuzzy sets and Systems, 116(2), 275-288.

Yong, D., \& Qi, L. (2005). A TOPSIS-based centroid-index ranking method of fuzzy numbers and its application in decision-making. Cybernetics and Systems: An International Journal, 36(6), 581-595.

Zadeh, L. A. (1965). Fuzzy sets. Information and control, 8(3), 338-353.

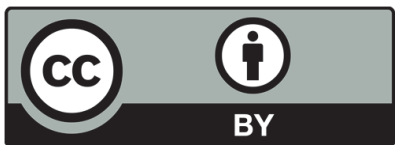

(C) 2019 by the authors; licensee Growing Science, Canada. This is an open access article distributed under the terms and conditions of the Creative Commons Attribution (CC-BY) license (http://creativecommons.org/licenses/by/4.0/). 